\title{
The Immunology of COVID-19: Your Questions Answered
}

\author{
By: Martin Profant (D)
}

\section{INTRODUCTION}

The COVID-19 pandemic, arguably one of the most significant challenges in recent history, continues to have a profound impact on everyone's lives. Beginning in late 2019, in a few months, SARS-CoV-2 has managed to spread to every corner of the globe. The virus has claimed the lives of over a million people and with global daily case counts approaching 600 000, there appears to be no end in sight ${ }^{1}$. Now with the second wave, the world is becoming desperate for an answer to this problem. The purpose of this article is to shed light on some of the questions that are key to understanding the immunology of COVID-19 and its medical implications.

\section{WHY IS COVID-19 SO DANGEROUS?}

Everyone has heard that COVID-19 is a deadly virus and that it has already cost the lives of many. Researchers understand the pathophysiology of the severe acute respiratory syndrome coronavirus 2 (SARS-CoV-2) quite well, despite the fact that this particular strain of virus arose only within the last year. Specifically, SARS-CoV-2 is a subtype of coronavirus known as a severe acute respiratory syndrome coronavirus $^{2}$. Many coronaviruses can cause disease in humans and animals, but the severe acute respiratory strains specifically infect the upper respiratory tract of humans ${ }^{2}$. Infection with SARS-CoV-2 is characterized by damage to lung tissue and aggressive inflammation ${ }^{2}$. Inflammation is a normal part of the immune response's attempt to contain and eliminate the infection, but excessive inflammation can be dangerous to human health ${ }^{2}$. COVID-19 (the disease caused by SARSCoV-2) can be deadly in severe cases by causing acute respiratory distress syndrome (ARDS) which can result in respiratory failure ${ }^{2}$. This is the leading cause of COVID-19 related deaths ${ }^{2}$. Excessive inflammation occurs because the body's immune response is trying to contain the infection and runs into overdrive; while the infection cannot be cleared because the SARSCoV-2 virus avoids immune detection (more on this later), the result of which is damaged lung tissue ${ }^{2}$. Combining the inflammatory damage with the damage the virus itself can create in infected tissue, the alveoli of the lungs can be severely impaired leading to an inability to perform life giving gas exchange ${ }^{3}$. The other issue with excessive inflammation is that it can lead to a cytokine storm which happens when the body's immune system releases too many cytokines (immune signalling molecules) rapidly ${ }^{3}$. This results in even more inflammation and can lead to multi-organ failure and subsequent death ${ }^{3}$. So COVID-19, is very dangerous and most often kills not because of the virus itself, but because of a dysfunctional immune response to the infection.

\section{HOW DOES SARS-COV-2 INFECT CELLS?}

The mechanism behind SARS-CoV-2 infection is quite simple. SARS-CoV-2 enters the respiratory tract of a human through the exposure to respiratory droplets from an infected individual. The virus particle then migrates to the lungs and uses the spike proteins on its surface, to bind to the angiotensin-converting enzyme 2 (ACE2) ${ }^{4}$. To bind to ACE2, the spike proteins must be cleaved by TMSPRSS ${ }^{4}$. The virus particle then fuses with the cell membrane and injects its viral package into the host cell ${ }^{4}$. The viral package contains the viral genome of single stranded RNA which is translated into NSPs (non-structural proteins) which along with the RNA genome, migrate to the endoplasmic reticulum of the cell ${ }^{4}$. There, the RNA genome is replicated, transcribed, and translated into proteins within the double membrane vesicles (another cellular organelle), which bud off from the endoplasmic reticulum ${ }^{4}$. The virus assembles within these vesicles, is released from the cell (killing it in the process), and afterwards the virus proceeds to infect other cells ${ }^{4}$.

\section{HOW DOES THE INNATE IMMUNE RESPONSE REACT TO SARS-COV-2?}

Upon infection of host cells with SARS-CoV-2, the innate immune response, which is the first line of 


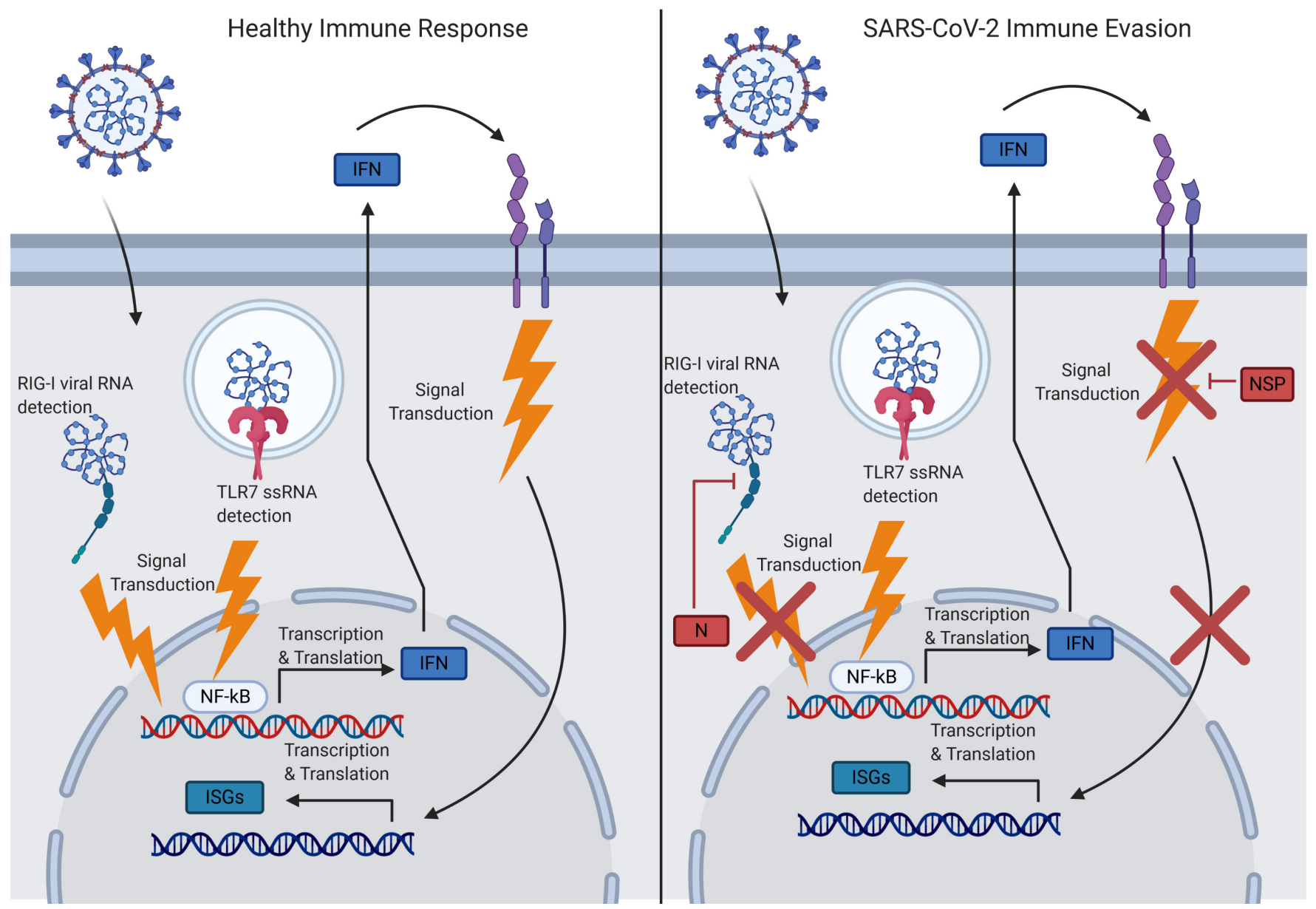

Figure 1. The healthy immune response to the SARS-CoV-2 virus is diagramed on the left and the mechanisms used by the virus to evade the immune response are shown on the right. The proteins in red represent SARS-CoV-2 viral proteins. The $\mathrm{N}$ protein causes degradation of the RIG-I receptor (through ubiquitination) and prevents it from detecting the virus' RNA genome. The NSP protein inhibits signal transduction from the interferon receptors and thus prevents the cells anti-viral response. Figure created with BioRender.

defense against any pathogen, kicks in. Innate immune cells detect the infection by recognizing pathogen structures called pathogen associated molecular patterns (PAMPs), using their pattern recognition receptors (PRRs) ${ }^{5}$. Specifically, in the case of SARSCoV-2 the RNA genome of the virus is detected by PRRs called TLR7 and RIG-I ${ }^{5}$. This receptor-ligand interaction leads to downstream signalling and the secretion of cytokines ${ }^{5}$. The cytokines released include interferons which heighten neighbouring cells' antiviral defenses, and production of proinflammatory cytokines (such as TNF- $\alpha, \mathrm{IL}-1, \mathrm{IL}-6$ ) that alert the rest of the immune system to the infection ${ }^{5}$. These signals recruit other immune cells like neutrophils and macrophages to arrive at the site of infection and destroy the virus particle ${ }^{5}$. Dendritic cells are also recruited which collect antigens from the virus and present them to CD4+ $\mathrm{T}$ cells which then coordinate the adaptive immune response ${ }^{5}$.

\section{WHY IS SARS-COV-2 NOT ELIMINATED BY THE IMMUNE SYSTEM?}

Part of the reason why SARS-CoV-2 is such a successful pathogen, is because of its ability to evade the immune system which it does in a number of ways. First, it avoids PRR detection by hiding its RNA genome in endoplasmic reticulum vesicles ${ }^{6}$. The endoplasmic reticulum does not contain PRRs, therefore, the foreign viral RNA cannot be detected ${ }^{6}$. The virus even goes one step further by degrading the PRR RIG-I, thereby preventing RIG-I from detecting the viral RNA. Recall that TLR7 and RIG-I, upon detecting viral PAMPs, initiate a signalling cascade that leads to the activation of the innate immune response ${ }^{6}$. SARS-CoV-2 has evolved to interfere with those signalling pathways and prevent the signal from being transmitted and the innate immune response from being activated ${ }^{6}$. The second main mechanism that SARS-CoV-2 uses to 
evade the immune system is by inhibiting interferon effectiveness ${ }^{6}$. As mentioned above, interferon is an important cytokine that enhances antiviral defenses in cells. First, the viral NSPs block the production of interferons by preventing transcription of their genes ${ }^{6}$. Furthermore, NSPs inhibit the signalling of the interferon receptors in neighbouring cells ${ }^{6}$. This prevents the neighbouring cells from receiving the message to begin their antiviral defenses. The end result is that the innate immune response cannot be effectively initiated and it is unable to contain the spread of the virus in the body. Note that SARS-CoV-2 only evades a part of the innate immune response (interferon pathway) and the rest of the immune system can contain the infection in the majority of cases.

\section{WHY ARE SOME COVID-19 CASES MORE SEVERE THAN OTHERS?}

It has become clear that COVID-19 affects people differently. Some people are asymptomatic, while others fight for their life in the ICU. According to the WHO $80 \%$ of infections are mild, $15 \%$ are severe, and $5 \%$ are critical (requiring ventilation) ${ }^{7}$. What is responsible for the differences in case severity, is a difficult question since there are many different factors which determine case severity, but some researchers have identified a few key mechanisms that may be responsible. One factor is how well the patient's immune system can mount a defense against the virus. A dysfunctional immune response can lead to a more severe case of COVID-193. Researchers found that two main factors determine whether the patient will elicit a dysfunctional immune response: the presence of autoantibodies and the patient's genetics ${ }^{8}$. Some patients have autoantibodies that attack the patient's own immune system and prevent it from effectively clearing the infection. Others have genetic mutations that make them unable to elicit an effective immune response against SARS-CoV-2 ${ }^{8}$. Another key factor in determining case severity is age. Older people have weakened immune systems and thus cannot combat the infection as well. In addition researchers have found that older people have more ACE2 expressed in their upper respiratory tract and since ACE2 is used by the virus to infect cells, more ACE2 receptors means a more severe SARS-CoV-2 infection? ${ }^{9}$.

\section{WHAT IS PASSIVE IMMUNIZATION?}

Passive immunization is intended to be a very short term and quick way for patients and healthcare workers to gain temporary immunity to the virus. The main form of passive immunization is convalescent plasma therapy, where the plasma from the blood of a recovered patient and is injected plasma into an afflicted patient ${ }^{10}$. The plasma contains antibodies that target the virus and will provide some short term immunity to the afflicted patient ${ }^{10}$. This treatment is termed passive because it does not activate the patient's immune system, instead the antibodies themselves confer immunity by binding to the virus and preventing it from infecting cells ${ }^{5}$. In terms of COVID-19, a small scale trial demonstrated that convalescent plasma therapy can be effective against the SARS-CoV-2 virus ${ }^{10}$; however the disadvantages are that the immunity is temporary and in severe cases it is not be enough to overcome the infection.

\section{WHAT ARE IMMUNOMODULATING DRUGS?}

Immunomodulating drugs, work by acting on the immune system to achieve symptom reduction. Oftentimes these drugs reduce inflammation in order to prevent ARDS and cytokine storms. The monoclonal antibody tocilizumab works by binding to the proinflammatory cytokine IL-6 to inhibit its signalling ability thereby reducing inflammation ${ }^{11}$. Tocilizumab is being tested in clinical trials for its efficacy in reducing the dangerous symptoms of inflammation in severe COVID-19 cases $^{11}$. Monoclonal antibodies are also being designed to specifically disrupt the entry of SARS-CoV-2 into host cells. Several research groups are developing monoclonal antibodies to inhibit the interaction between ACE2 and the virus' spike proteins, which is required for the virus to enter host cells ${ }^{12}$. There are some challenges associated with the use of monoclonal antibodies for COVID-19, particularly the fact that antibody production is difficult and expensive, therefore it is challenging to administer as a wide spread treatment ${ }^{12}$.

\section{DO ANTIVIRAL DRUGS WORKAS TREATMENT AGAINST COVID-19?}

Antiviral drugs have been discussed as a possible treatment for COVID-19. These drugs come in many different types from broad spectrum antivirals to antivirals meant to treat other infections like HIV or malaria. These drugs all have one thing in common: they target a process in the SARS-CoV-2 life cycle and disrupt it. For instance, the drug camostat mesylate works by inhibiting TMPRSS2 which is necessary for SARS-CoV-2 to enter the host cells ${ }^{2}$. Drugs like lopinavir and ritonavir along with ribavirin have been proposed to treat COVID-19, as some studies point to their ability to inhibit viral replication in the cell ${ }^{13}$. The Ebola drug remdesivir has been widely debated as a potential COVID-19 treatment because it can inhibit viral RNA synthesis ${ }^{13}$. Perhaps most famously are the anti-malarial drugs chloroquine and hydroxychloroquine which have many effects on the viral life cycle, but mainly work by inhibiting viral exocytosis (the process of viral escape from the cell) by raising the $\mathrm{pH}$ in lysosomes which are required for viral protein processing ${ }^{11}$. Studies suggest that all these drugs do have some positive effects on treating COVID-19 and do reduce recovery time and 


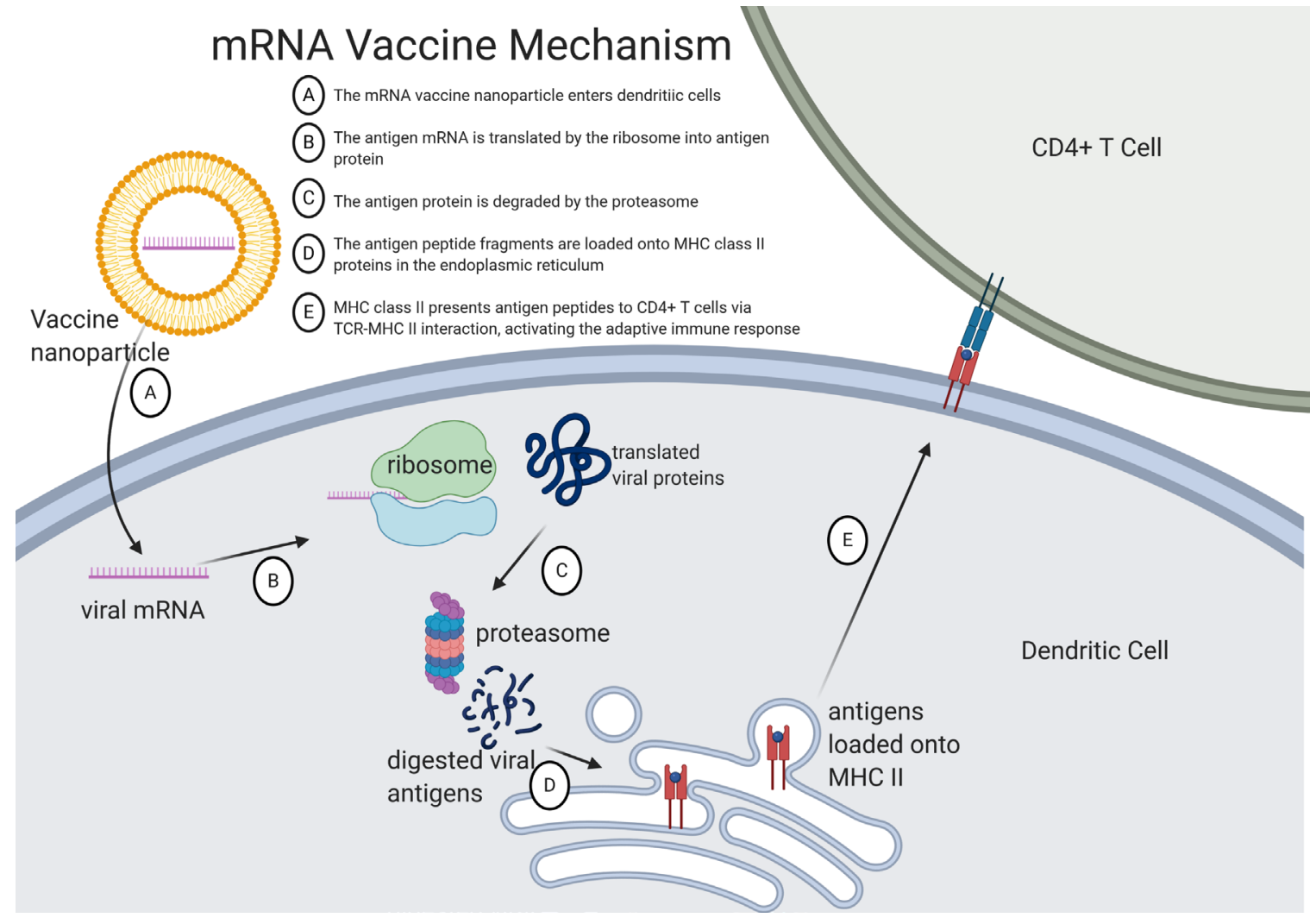

Figure 2. The mechanism of mRNA vaccination. Figure created with BioRender.

mortality, but more research must be done to establish clear conclusions ${ }^{13}$. However, none have been shown to be a magic bullet that is able to completely clear the infection within a few days on its own. Therefore, these drugs are probably useful for treating patients for the disease, but they are not a solution to the global pandemic.

\section{WHAT ARE MRNA VACCINES AND WHY ARE THEY SO EXCITING?}

mRNA vaccines are a novel form of nucleic acid vaccine. The mRNA which encodes the viral proteins is injected into the body where it is taken up by dendritic cells (antigen presenting immune cells) that translate the mRNA into protein and present it on their MHC class II (antigen presenting molecule) to stimulate $\mathrm{T}$ cells and the subsequent adaptive immune responses ${ }^{14}$. The advantage of this approach is that the adaptive immune response is more directly activated which improves the efficacy of the vaccine. These mRNA vaccines can be developed extremely quickly since all they require is the genetic sequence of the viral protein to be used as an antigen ${ }^{11}$. They may also be safer since only part of the virus is being produced rather than injecting an entire attenuated virus ${ }^{14}$. The disadvantages are that mRNA is fragile and thus storage and delivery of the vaccine is a challenge ${ }^{14}$. Also, this technology is unproven and has yet to be used against any disease thus there could be unknown risks ${ }^{15}$. One particular vaccine candidate to note is the mRNA vaccine BNT162a1/b1/ b2/c2 developed by BioNTech and Pfizer². Recently the companies announced that this mRNA vaccine candidate achieved over $90 \%$ effectiveness in its clinical trial ${ }^{16}$. This vaccine candidate utilizes the same mRNA technology discussed earlier and uses the SARS-CoV-2 spike protein as the antigen of choice ${ }^{16}$. The companies say they can produce 50 million doses of the vaccine by the end of 2020 and up to 1.3 billion by early 2021 which makes this the most promising vaccine candidate to date ${ }^{16}$. The disadvantage of the BioNTech vaccine is that it requires storage at $-80^{\circ} \mathrm{C}$ making distribution across the world quite challenging ${ }^{16}$. This data is strong evidence for the use of the spike protein as an antigen and for the efficacy of mRNA vaccines $^{12}$. There are still nearly 50 other vaccines in clinical trials and the data from them will be available in the next several months ${ }^{16}$. There is reason to be hopeful, but being a good scientist means approaching this data with caution and reasonable skepticism.

\section{CONCLUSION: WHAT DOES THIS ALL MEAN?}

The immunology of COVID-19 is complex and foggy because at times the immune system can be a formidable foe or a great ally. Hopefully, you better understand the mechanisms that underlie SARS-CoV2's entry into the body, how it causes disease, and 
how different treatments utilize various mechanisms to target and eliminate the virus and its symptoms. While we wait for science to find an effective weapon against this disease, remember that everyone has a role to play. Embracing social distancing and good hygiene, having the awareness and responsibility to limit your contact with others are all key measures to slow the spread of the virus. After all, prevention is the best medicine.

Martin Profant is a student at the University of Toronto and a Staff Writer for the JULS. Please address correspondence to: martin.profant@mail.utoronto.ca

\section{COMPETITING INTERESTS}

No competing interests declared.

Received: November 22, 2020

Accepted: January 4, 2021

Published online: January 22, 2021

\section{REFERENCES}

1. John Hopkins University . COVID-19 Map. Johns Hopkins Coronavirus Resource Center (2020). Available at: https:// coronavirus.jhu.edu/map.html. (Accessed: 10th November 2020)

2. Tay, M. Z., Poh, C. M., Rénia, L., Macary, P. A. \& Ng, L. F. P. The trinity of COVID-19: immunity, inflammation and intervention. Nature Reviews Immunology 20, 363-374 (2020).

3. Vabret, N. et al.Immunology of COVID-19: Current State of the Science. Immunity 52, 910-941 (2020).

4. V'Kovski, P., Kratzel, A., Steiner, S., Stalder, H. \& Thiel, V. Coronavirus biology and replication: implications for SARS-CoV-2. Nature Reviews Microbiology (2020). doi:10.1038/s41579-02000468-6

5. Murphy, K. M., Weaver, C. \& Janeway, C. Janeway's immunobiology. (Garland Science/Taylor \& Francis Group, 2017).

6. Taefehshokr, N., Taefehshokr, S., Hemmat, N. \& Heit, B. Covid-19: Perspectives on Innate Immune Evasion. Frontiers in Immunology 11, (2020).

7. World Health Organization. Coronavirus disease 2019(COVID-19) Situation Report - 46. WHO(2020). Available at: https://www.who. int/docs/default-source/coronaviruse/situation-reports/20200306sitrep-46-covid-19.pdf?sfvrsn=96b04adf_4\#: :text=For $\% 20$ COVID\%2D19\%2C, infections\%2C\%20requiring\%20ventilation. (Accessed: 14th November 2020)

8. NIH. Scientists discover genetic and immunologic underpinnings of some cases of severe COVID-19. National Institutes of Health(2020). Available at: https://www.nih.gov/news-events/newsreleases/scientists-discover-genetic-immunologic-underpinningssome-cases-severe-covid-19. (Accessed: 14th November 2020)

9. Bunyavanich, S., Do, A. \& Vicencio, A. Nasal Gene Expression of Angiotensin-Converting Enzyme 2 in Children and Adults. JAMA 323, 2427 (2020).

10. Abraham, J. Passive antibody therapy in COVID-19. Nature Reviews Immunology 20, 401-403 (2020).

11. Zhong, J., Tang, J., Ye, C. \& Dong, L. The immunology of
COVID-19: is immune modulation an option for treatment? The Lancet Rheumatology 2, (2020).

12. Jahanshahlu, L. \& Rezaei, N. Monoclonal antibody as a potential anti-COVID-19. Biomedicine \& Pharmacotherapy 129, 110337 (2020).

13. Ali, M. J. et al.Treatment Options for COVID-19: A Review. Frontiers in Medicine 7, (2020).

14. Pardi, N., Hogan, M. J., Porter, F. W. \& Weissman, D. mRNA vaccines - a new era in vaccinology. Nature Reviews Drug Discovery 17, 261-279 (2018).

15. Dong, Y. et al. A systematic review of SARS-CoV-2 vaccine candidates. Signal Transduction and Targeted Therapy 5, (2020).

16. Cohen, J. Champagne and questions greet first data showing that a COVID-19 vaccine works. Science (2020). Available at: https://www.sciencemag.org/news/2020/11/champagne-andquestions-greet-first-data-showing-covid-19-vaccine-works. (Accessed: 20th November 2020) 